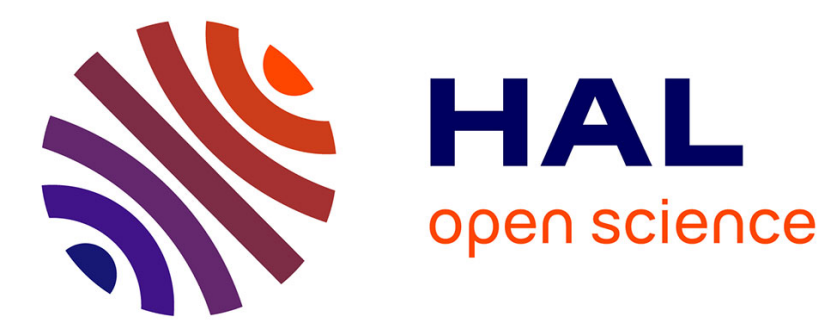

\title{
Projective structure and holonomy in general relativity
}

G S Hall, D P Lonie

\section{To cite this version:}

G S Hall, D P Lonie. Projective structure and holonomy in general relativity. Classical and Quantum Gravity, 2011, 28 (8), pp.83101. 10.1088/0264-9381/28/8/083101 . hal-00690071

\section{HAL Id: hal-00690071 https://hal.science/hal-00690071}

Submitted on 21 Apr 2012

HAL is a multi-disciplinary open access archive for the deposit and dissemination of scientific research documents, whether they are published or not. The documents may come from teaching and research institutions in France or abroad, or from public or private research centers.
L'archive ouverte pluridisciplinaire HAL, est destinée au dépôt et à la diffusion de documents scientifiques de niveau recherche, publiés ou non, émanant des établissements d'enseignement et de recherche français ou étrangers, des laboratoires publics ou privés. 


\title{
Projective Structure and Holonomy in General Relativity
}

\author{
G S Hall ${ }^{1}$ and D P Lonie $^{2}$ \\ ${ }^{1}$ Institute of Mathematics, University of Aberdeen, Aberdeen, AB24 3UE, Scotland, \\ $\mathrm{UK}$ \\ ${ }^{2}$ School of Computing, The Robert Gordon University, Aberdeen, AB25 1HG, \\ Scotland, U.K. \\ E-mail: g.hall@abdn.ac.uk, dplonie@gmail.com
}

\begin{abstract}
This paper presents a study of the situation when two space-times admit the same (unparametrised) geodesics, that is, when they are projectively related. The solution is based on the curvature class and the holonomy type of a space-time and it transpires that all holonomy possibilities can be solved except the most general one and that the consequence of two space-times being projectively related leads, in many cases, to their associated Levi-Civita connections being identical. Some results are also given regarding the general case. It is also shown that the holonomy types of projectively related space-times are very closely related. The theory is then applied, with Einsteins principle of equivalence in mind, to "generic" space-times.
\end{abstract}

PACS numbers: 04.20.-q, 02.40.-k, 02.40.Ky

Submitted to: Class. Quantum Grav. 


\section{Introduction}

In Newton's theory of gravity, the second and third laws suggest that, by an appropriate choice of units, one may equate the active and passive gravitational masses and the inertial mass of any material particle. This reveals the existence of a well-defined acceleration field at each space-time point which any such (free) particle under the action of a gravitational field must obey independently of its mass, make-up and velocity. In Einstein's general theory of relativity, such a behaviour of particles led, once the concept of a space-time manifold equipped with a Lorentz metric had been formulated, to the assumption that the paths of such particles were timelike geodesics with respect to the Levi-Civita connection of the metric.

This Newton-Einstein principle of equivalence leads to the following mathematical question. Suppose that space-time is described, as in general relativity, by a 4dimensional (smooth, connected, hausdorff) manifold $M$ admitting a (smooth) Lorentz metric $g$ of signature $(-1,+1,+1,+1)$ and associated Levi-Civita connection $\nabla$. Suppose that $g^{\prime}$ is another (smooth) Lorentz metric on $M$ with Levi-Civita connection $\nabla^{\prime}$ such that the (unparametrised) geodesics of $\nabla$ and $\nabla^{\prime}$ coincide. What is the relationship between $\nabla$ and $\nabla^{\prime}$ and between $g$ and $g^{\prime}$ ? Under these conditions $\nabla$ and $\nabla^{\prime}$ (or $g$ and $g^{\prime}$, or $(M, g)$ and $\left.\left(M, g^{\prime}\right)\right)$ are said to be projectively related. Thus the above problem is to determine the relationship between $\nabla$ and $\nabla^{\prime}$ and between $g$ and $g^{\prime}$ for projectively related space-times. In the event that this condition forces the result $\nabla^{\prime}=\nabla, \nabla^{\prime}$ and $\nabla$ (or $g$ and $g^{\prime}$ ) will be referred to as trivially projectively related (or affinely related). It will also be useful on many occasions to have the concept of local projective relatedness. Let $(M, g)$ be a space-time and $U$ a connected open submanifold of $M$. A metric $g^{\prime}$ on $U$ (respectively, its Levi-Civita connection $\nabla^{\prime}$ ) will be said to be (locally) projectively related (on $U$ ) to $g$ (respectively, to its Levi-Civita connection) if the space-time $\left(U, g^{\prime}\right)$ is projectively related in the above sense to the space-time $(U, g)$ consisting of the manifold $U$ with the metric $g$ restricted to it. This reduces to the previous definition if $U=M$ and then this situation is sometimes referred to as global projective relatedness. There is a technical point here in that if the original manifold $M$ is specified, the calculations performed on $U$ are somewhat indifferent to $M$ and the metric $g$ so found may not be extendible to $M$. In such a case, $M$ may have to be redefined as (restricted to) some open submanifold $V$ of $M$ with $U \subset V$ and on which $g$ is defined. This will be understood in what is to follow. In the event that $(M, g)$ and $\left(M, g^{\prime}\right)$ are projectively related and if, as a consequence, $\nabla=\nabla^{\prime}$, holonomy theory can be used to find the relationship between $g$ and $g^{\prime}$ [1]. [It is remarked here that there are solutions to the above type of problem with $g$ and $g^{\prime}$ of differing signature. Here, however, it will assumed that $g$ and $g^{\prime}$ are each of Lorentz signature.] It is convenient to introduce the definition that a space-time is non-flat if its curvature tensor, Riem, does not vanish over any non-empty open subset of $M$ (and is not to be confused with not flat, which means exactly that). 


\section{Mathematical Preliminaries}

In order to solve the problem raised in the last section let $(M, g)$ be a space-time with Levi-Civita connection $\nabla$ and curvature tensor Riem. A solution of the problem in the general case seems to be rather difficult and the idea is to consider certain special cases in order to make the problem more tractable. For the important situation when $(M, g)$ is a vacuum space-time a very strong result is available and has been discovered, to a large extent independently, in $[2,3,4,5]$. This result says that if $(M, g)$ is vacuum and not flat and if a space-time $\left(M, g^{\prime}\right)$ with Levi-Civita connection $\nabla^{\prime}$ is projectively related to it then $\nabla=\nabla^{\prime}$ (and so $\left(M, g^{\prime}\right)$ is also vacuum) and, with exclusion of the case when $(M, g)$ is a $p p$-wave, $g^{\prime}=c g$ for some constant $c[3,4]$. Thus, with $c$ taken positive, $g$ and $g^{\prime}$ are the same up to "units of measurement". [In fact a little more can be said, see e.g. [4].] In another important class of metrics, the FRWL cosmological metrics, one can still solve the problem, at least in the local sense mentioned earlier, but now $\nabla$ and $\nabla^{\prime}$ need not be equal but the relation between $g$ and $g^{\prime}$ can still be found ([6]-see also $[7,8])$.

A more general attack on the problem was initiated in [9] where $(M, g)$ was first classified according to its holonomy group type and curvature class and the projective relatedness problem was then studied for each such type and class. In this paper a brief description of the completion of this study is given and where it will be shown that the problem can essentially be solved for all holonomy types except the most general one. The full details are somewhat lengthy and may be of limited interest to relativists and will be published elsewhere ([9] and [11]) but summarised here in a form which it is hoped will be useful to those working in Einsteins theory. The possible holonomy groups for a space-time (more precisely for its connection) may be classified into 15 mutually exclusive and exhaustive types and are labelled, following [10], by the symbols $R_{1}, \ldots R_{15}$ with $R_{1}$ being the trivial flat space-time case and $R_{15}$ the most general case (and $R_{5}$ cannot occur for a space-time). Since full details of this classification scheme are available [1] (and a summary has been given in $[9,11]$ ) no further review need be given here. The holonomy type of a space-time is a global statement about it and it is useful to introduce a supplementary (pointwise) classification in $M$ determined by the curvature tensor Riem with components $R_{b c d}^{a}$. This classification has also been given in detail in [1] (and summarised in [9, 11]) but will be partially repeated here in order to clarify notation. The remainder of the paper is devoted to a summary of the main results and techniques regarding projective relatedness, to demonstrating the (close) relationship between the holonomy types of projectively related metrics, to a uniqueness theorem which exists in the more complicated $R_{9}$ and $R_{14}$ cases of section 4 , to a "rigidity" result for projective relatedness which exists for what might be called "generic" space-times and to an application of these results to the study of projective symmetry in space-times. The last four of these are believed to be new.

Let $m \in M$ and consider the curvature map $f$ which is the linear map from the vector space of bivectors (skew-symmetric second order tensors) at $m$ to itself and given 
by $f: F^{a b} \rightarrow R^{a b}{ }_{c d} F^{c d}$. The rank of the map $f$ is referred to as the curvature rank (of Riem) at $m$ [1]. Let $B_{m}$ denote the range space of $f$ at $m$ so that $\operatorname{dim} B_{m}$ equals the curvature rank at $m$ and is $\leq 6$. This leads to a convenient algebraic classification of Riem at $m$ into five mutually exclusive and disjoint curvature classes. For this it is convenient to denote a simple bivector $F^{a b}=p^{a} q^{b}-q^{a} p^{b}$ for $p, q \in T_{m} M$ by $p \wedge q$. (It is remarked for later purposes that a non-simple bivector $F$ may be written as the sum of two simple bivectors whose blades are, respectively, timelike and spacelike and mutually orthogonal. These blades, which are uniquely determined by $F$, are referred to as the canonical pair of blades of $F$.) On occasions, round and square brackets will be used to denote the symmetrisation and skew-symetrisation of the indices they enclose.

Class A This covers all possibilities not covered by classes B, C, D and O below. For this class, the curvature rank at $m$ is $2,3,4,5$ or 6 .

Class B This occurs when $\operatorname{dim} B_{m}=2$ and when $B_{m}$ is spanned by a timelike-spacelike pair of simple bivectors with orthogonal blades (chosen so that one is the dual of the other). In this case, one can choose a null tetrad $l, n, x, y \in T_{m} M$ such that these bivectors are $F=l \wedge n$ and $\stackrel{*}{F}=x \wedge y$, so that $F$ is timelike and $\stackrel{*}{F}$ is spacelike and then (using the algebraic identity $R_{a[b c d]}=0$ to remove cross terms) one has, at $m$,

$$
R_{a b c d}=\alpha F_{a b} F_{c d}+\beta \stackrel{*}{F}_{a b} \stackrel{*}{F}_{c d}
$$

for $\alpha, \beta \in \mathbb{R}, \alpha \neq 0 \neq \beta$.

Class $\mathbf{C}$ In this case $\operatorname{dim} B_{m}=2$ or 3 and $B_{m}$ may be spanned by independent simple bivectors $F$ and $G$ (or $F, G$ and $H$ ) with the property that there exists $0 \neq r \in T_{m} M$ such that $r$ lies in the blades of $\stackrel{*}{F}$ and $\stackrel{*}{G}$ ( or $\stackrel{*}{F}, \stackrel{*}{G}$ and $\left.\stackrel{*}{H}\right)$. Thus $F_{a b} r^{b}=G_{a b} r^{b}\left(=H_{a b} r^{b}\right)=0$ and $r$ is then unique up to a multiplicative non-zero real number.

Class D In this case $\operatorname{dim} B_{m}=1$. If $B_{m}$ is spanned by the bivector $F$ then, at $m$,

$$
R_{a b c d}=\alpha F_{a b} F_{c d}
$$

for $0 \neq \alpha \in \mathbb{R}$ and $R_{a[b c d]}=0$ implies that $F_{a[b} F_{c d]}=0$ from which it may be checked that $F$ is necessarily simple.

Class O In this case Riem vanishes at $m$.

This classification is, of course, pointwise and may vary over $M$. A space-time $(M, g)$ (or some subset of it) which has the same curvature class at each point of $M$ is said to be of that class. The subset of $M$ consisting of points at which the curvature class is $\mathbf{A}$ is an open subset of $M([1], \mathrm{p}$ 393) and the analogous subset arising from the class $\mathbf{O}$ is closed (and has empty interior in the manifold topology of $M$ if $(M, g)$ is non-flat). It is important in what is to follow that the equation $R_{a b c d} k^{d}=0$ at $\mathrm{m}$ has no non-trivial solutions for $k \in T_{m} M$ if the curvature class at $m$ is $\mathbf{A}$ or $\mathbf{B}, a$ unique independent solution (the vector $r$ above) if the curvature class at $m$ is $\mathbf{C}$ and 
two independent solutions if the curvature class at $m$ is $\mathbf{D}$ (and which span the blade of $\stackrel{*}{F}$ in $(2))$. If $\operatorname{dim} B_{m} \geq 4$ the curvature class at $m$ is necessarily $\mathbf{A}$.

The following result will be useful in what is to follow and the details and proof can be found in [1].

Theorem 1 Let $(M, g)$ be a space-time, let $m \in M$ and let $h$ be a non-zero second order, symmetric, type (0,2) (not necessarily non-degenerate) tensor at $m$ satisfying $h_{a e} R_{b c d}^{e}+h_{b e} R_{a c d}^{e}=0$.

(i) If the curvature class of $(M, g)$ at $m$ is $\mathbf{D}$ and $u, v \in T_{m} M$ span the 2-space at $m$ orthogonal to $F$ in (2) (that is, $u \wedge v$ is the blade of $F$ ) there exists $\phi, \mu, \nu, \lambda \in \mathbb{R}$ such that, at $m$,

$$
h_{a b}=\phi g_{a b}+\mu u_{a} u_{b}+\nu v_{a} v_{b}+\lambda\left(u_{a} v_{b}+v_{a} u_{b}\right)
$$

(ii) If the curvature class of $(M, g)$ at $m$ is $\mathbf{C}$ there exists $r \in T_{m} M$ (the vector appearing in the above definition of class $\mathbf{C}$ ) and $\phi, \lambda \in \mathbb{R}$ such that, at $m$,

$$
h_{a b}=\phi g_{a b}+\lambda r_{a} r_{b}
$$

(iii) If the curvature class of $(M, g)$ at $m$ is $\mathbf{B}$ there exists a null tetrad $l, n, x, y$ (that appearing in the above definition of class $\mathbf{B}$ ) and $\phi, \lambda \in \mathbb{R}$ such that, at $m$ (and making use of the associated completeness relation),

$$
h_{a b}=\phi g_{a b}+\lambda\left(l_{a} n_{b}+n_{a} l_{b}\right)=(\phi+\lambda) g_{a b}-\lambda\left(x_{a} x_{b}+y_{a} y_{b}\right)
$$

(iv) If the curvature class of $(M, g)$ at $m$ is $\mathbf{A}$ there exists $\phi \in \mathbb{R}$ such that, at $m$,

$$
h_{a b}=\phi g_{a b}
$$

[Thus if $g^{\prime}$ is another metric on $M$ whose curvature tensor Riem' equals the curvature tensor Riem of $g$ everywhere on $M$ then the conditions of this theorem are satisfied for $h=g^{\prime}(m)$ at each $m \in M$ and so the conclusions also hold except that now one must add the restriction $\phi \neq 0$ in each case to preserve the non-degeneracy of $g^{\prime}$ at $m$ and maybe some restrictions on $\phi, \mu, \nu$ and $\lambda$ if the signature of $g^{\prime}$ is prescribed. If $(M, g)$ is of class $\mathbf{A},(6)$ gives $g^{\prime}=\phi g$ for some function $\phi: M \rightarrow \mathbb{R}$ and the Bianchi identity may be used to show that $\phi$ is constant on $M[1]$.

\section{Projective Relatedness}

It is natural to think that the problem of projective relatedness should be related in some way to holonomy theory and it is the object of this paper to show that the problem of determining all space-times projectively related (or locally projectively related) to a given space-time $(M, g)$ can essentially be solved when the connection $\nabla$ of $(M, g)$ is of any holonomy type except the most general one $R_{15}$ and that some progress can be made even in this latter case. Some of the proofs are lengthy and technical and an attempt will be made to keep such details to a minimum.

So suppose that $(M, g)$ is projectively related to $\left(M, g^{\prime}\right)$ with $g^{\prime}$ of Lorentz signature (in fact it will be clear from the calculations below that the signature of $g^{\prime}$, projectively 
related to $g$, need not always be Lorentz but this will not be discussed further here). Then $M$ necessarily admits a smooth global 1-form $\psi$ such that, in any coordinate domain, the Christoffel symbols $\Gamma$ and $\Gamma^{\prime}$ of $\nabla$ and $\nabla^{\prime}$, respectively, satisfy $[12,13,14]$

$$
\Gamma_{b c}^{a}-\Gamma_{b c}^{a}=\delta_{b}^{a} \psi_{c}+\delta_{c}^{a} \psi_{b}
$$

and, conversely, if (7) holds in any coordinate domain for some global 1-form $\psi,(M, g)$ and, $(M, g)$ and $\left(M, g^{\prime}\right)$ are projectively related. Since $\nabla$ and $\nabla^{\prime}$ are metric connections $\psi$ is an exact 1-form on $M$ (see, e.g. [14]) and so $\psi=d \chi$ for some smooth function $\chi$ on $M$. Equation (7) can, by using the identity $\nabla^{\prime} g^{\prime}=0$, be written in the equivalent form

$$
g_{a b ; c}^{\prime}=2 g_{a b}^{\prime} \psi_{c}+g_{a c}^{\prime} \psi_{b}+g_{b c}^{\prime} \psi_{a}
$$

where a semi-colon denotes a covariant derivative with respect to $\nabla$. Equation (7) reveals a relation between the type $(1,3)$ curvature tensors Riem and Riem ${ }^{\prime}$ of $\nabla$ and $\nabla^{\prime}$, respectively, given by

$$
R_{b c d}^{\prime a}=R_{b c d}^{a}+\delta_{d}^{a} \psi_{b c}-\delta_{c}^{a} \psi_{b d} \quad\left(\Rightarrow R_{a b}^{\prime}=R_{a b}-3 \psi_{a b}\right)
$$

where $\psi_{a b} \equiv \psi_{a ; b}-\psi_{a} \psi_{b}=\psi_{b a}$ and where $R_{a b} \equiv R_{a c b}^{c}$ and $R_{a b}^{\prime} \equiv R_{a c b}^{\prime c}$ are the Ricci tensor components of $\nabla$ and $\nabla^{\prime}$, respectively.

Thus the problem considered has been reduced to the solving of (8) for $g^{\prime}$ and $\psi$. However, the problem can, to some extent, be simplified by adopting the Sinjukov transformation ([15], see also $[4,5,8,9])$. This technique involves introducing another non-degenerate second order symmetric tensor $a$ and another 1 -form $\lambda$ to replace $g^{\prime}$ and $\psi$ and which are defined in terms of them by

$$
a_{a b}=e^{2 \chi} g^{\prime c d} g_{a c} g_{b d} \quad \lambda_{a}=-e^{2 \chi} \psi_{b} g^{\prime b c} g_{a c}\left(\Rightarrow \lambda_{a}=-a_{a b} \psi^{b}\right)
$$

where an abuse of notation has been used in that $g^{\prime a b}$ denotes the contravariant components of $g^{\prime}$ (and not the tensor $g_{a b}^{\prime}$ with indices raised using $g$ ) so that $g_{a c}^{\prime} g^{\prime c b}=\delta_{a}{ }^{b}$. Then (10) may be inverted to give

$$
g^{\prime a b}=e^{-2 \chi} a_{c d} g^{a c} g^{b d} \quad \psi_{a}=-e^{-2 \chi} \lambda_{b} g^{b c} g_{a c}^{\prime}
$$

The condition (8) for projective relatedness is now, from (10) and (11), equivalent to Sinjukov's equation [15]

$$
a_{a b ; c}=g_{a c} \lambda_{b}+g_{b c} \lambda_{a}
$$

from which it easily follows that $\lambda$ is an exact 1-form, being the global gradient of $\frac{1}{2} a_{a b} g^{a b}$. The object now becomes to solve (12) for $a$ and $\lambda$. With $a$ and $\lambda$ thus found, one first defines a type (2.0) tensor $a^{-1}$ on $M$ which is, at each $m \in M$, the inverse matrix of $a\left(a_{a c} a^{-1 c b}=\delta_{a}^{b}\right)$. Then one defines a related type $(0,2)$ tensor on $M$ by $a_{a b}^{-1}=g_{a c} g_{b d} a^{-1 c d}$. Finally, one defines a global function $\chi=\frac{1}{2} \log \left|\left(\frac{\operatorname{det} g}{\operatorname{det} a}\right)\right|$ and a global exact 1-form $\psi \equiv d \chi$ on $M$. Then $g_{a b}^{\prime}=e^{2 \chi} a_{a b}^{-1}$, which is a global metric on $M$, and $\psi$ together satisfy (11) and constitute the required solution of (8) on $M$. 
It is useful here to note that, using (12), and applying the Ricci identity to $a$, one finds (see, e.g. [5])

$$
\left(a_{a b ; c d}-a_{a b: d c}=\right) a_{a e} R_{b c d}^{e}+a_{b e} R_{a c d}^{e}=g_{a c} \lambda_{b d}+g_{b c} \lambda_{a d}-g_{a d} \lambda_{b c}-g_{b d} \lambda_{a c}
$$

where $\lambda_{a b}=\lambda_{a ; b}=\lambda_{b a}$. This leads to the following lemma, part of which is a special case of a result in [3] and for which a definition is required. A (real) bivector $F$ at $m \in M$ is called a (real) eigenbivector of Riem (or of the curvature map $f$ ) at $m$ if $R^{a b}{ }_{c d} F^{c d}=\alpha F^{a b}$ at $m$ and $\alpha \in \mathbb{R}$ is called the associated eigenvalue.

Lemma 1 Let $(M, g)$ and $\left(M, g^{\prime}\right)$ be space-times with $g$ and $g^{\prime}$ projectively related.

(i) Suppose at $m \in M$ that $F$ is a (real) eigenbivector of Riem of $(M, g)$ with zero eigenvalue (so that $F$ is in the kernel, ker $f$, of the map $f$ ). Then the blade of $F$ (if $F$ is simple) or each of the canonical (orthogonal, timelike/spacelike) pair of blades of $F$ (if $F$ is non-simple) is an eigenspace of the symmetric tensor $\lambda_{a b}$ with respect to $g$ at $m$. (That is, if $p \wedge q$ is any of these blades $\left(p, q \in T_{m} M\right)$ there exists $\mu \in \mathbb{R}$ such that for any $\left.k \in p \wedge q, \lambda_{a b} k^{b}=\mu g_{a b} k^{b}\right)$.

(ii) Suppose ker $f$ is such that $T_{m} M$ is forced to be an eigenspace of $\lambda_{a ; b}$ at each $m^{\prime}$ in some connected open neighbourhood $U$ of $m$, then each of the following conditions are satisfied on $U$ for some $c \in \mathbb{R}$
(a) $\lambda_{a b}\left(=\lambda_{a ; b}\right)=c g_{a b}$
(b) $\lambda_{d} R_{a b c}^{d}=0$
(c) $a_{a e} R_{b c d}^{e}+a_{b e} R_{a c d}^{e}=0$

If (14) holds at each $m \in M$ either $\lambda$ vanishes identically on $M$ or it does not. In the latter case, whether $c$ is zero or not, any point of $M$ at which it does vanish is topologically isolated and the subset of such points constitutes a closed subset of $M$ with empty interior. Further, Riem vanishes on some neighbourhood of any such point. If $\lambda$ vanishes on some non-empty open subset of $M$ it vanishes on $M$.

The results in the last part of lemma 1 rely on the fact that $\lambda$ is either covariantly constant $(c=0)$ or a proper homothetic (co)vector field $(c \neq 0)$ with zero homothetic bivector on $U$ [1]. It is important to note here that the conditions of lemma 1 which lead to (14) hold at any $m \in M$ if $M$ is of holonomy type $R_{2}, R_{3}, R_{4}, R_{6}, R_{7}, R_{8}, R_{10}$, $R_{11}, R_{12}$ or $R_{13}$ or if it is of curvature class $\mathbf{B}, \mathbf{C}$ or $\mathbf{D}$ and is thus of wide applicability.

Let $S$ denote the set of all pairs $(a, \lambda)$ which are, together, solutions of (12) but without the restriction that $a$ is non-singular. Then with additon and scalar multiplication by real numbers defined for $(a, \lambda)$ and $(b, \mu) \in S$ and $\alpha \in \mathbb{R}$ by $(a, \lambda)+(b, \mu)=(a+b, \lambda+\mu)$ and $\alpha(a, \lambda)=(\alpha a, \alpha \lambda), S$ becomes a real vector space. Now one can show from (13) that $\lambda_{a}, \lambda_{; a}^{a}$ and $a_{a b}$ are controlled by a first order system of differential equations and hence any global solution for these quantites is uniquely determined by their values at any point of $M$ ([8], see also [11]). It can then be seen that the vector space $S$ is finite dimensional. [A special case of this result is the "rigidity" of the homothetic vector field in the above lemma (that is, if it vanishes over some nonempty open subset of $M$ it vanishes on $M$ ).] From this remark one has the following theorem. 
Theorem 2 Let $(M, g)$ be a space-time. If the pairs $(a, \lambda),(b, \mu) \in S$ and if there exists a non-empty open subset $U \subset M$ such that $b=a+\alpha g(\alpha \in \mathbf{R})$ on $U$ then $b=a+\alpha g$ and $\lambda=\mu$ on $M$. In particular, if $a=b$ on $U$ then $a=b$ and $\lambda=\mu$ on $M$ and so $(a, \lambda)=(b, \mu)$. Thus, if $M$ admits a non-empty, open subset $U$ such that the only solution of (12) on $U$ is $\lambda=0$ and $a=\alpha g(0 \neq \alpha \in \mathbf{R})$ then $\nabla=\nabla^{\prime}$ on $M$ (that is, the only solution of (12) on $M$ is $\lambda=0$ and $a=\alpha g$ ).

\section{Projective Relatedness and Holonomy}

The following theorem serves to concentrate attention onto the holonomy types $R_{9}$, $R_{14}, R_{15}$ and special subcases of the holonomy types $R_{10}, R_{11}$ and $R_{13}$. It is noted with regard to this result that, for the holonomy types $R_{10}, R_{11}$ and $R_{13}$, the curvature rank at any $m \in M$ is at most 3 .

\section{Theorem 3}

(i) Let $(M, g)$ be a space-time of holonomy type $R_{2}, R_{3}, R_{4}, R_{6}, R_{7}, R_{8}$ or $R_{12}$ which is projectively related to $\left(M, g^{\prime}\right)$. Then $\nabla=\nabla^{\prime}$ on $M$.

(ii) Let $(M, g)$ be a space-time of holonomy type $R_{10}, R_{11}$ or $R_{13}$ such that there exists $m \in M$ at which the curvature rank of $(M, g)$ is 2 or 3 (equivalently the curvature class at $m$ is $\mathbf{C})$. Then $\nabla=\nabla^{\prime}$ on $M$.

Since $\nabla=\nabla^{\prime}$ the relationship between $g$ and $g^{\prime}$ can be written down in each case [1].

To complete the solution for all holonomy types except $R_{9}, R_{14}$ and $R_{15}$ one needs only to consider the situation when $(M, g)$ is of holonomy type $R_{10}, R_{11}$ and $R_{13}$ and when the curvature rank is $\leq 1$ at each $m \in M$. If such is the case, and since $(M, g)$ is not flat, there exists $m \in M$ and hence an open neighbourhood $U$ of $m$ on which Riem is everywhere of curvature rank 1 . Again the conclusions of the lemma hold on $M$ and if $(M, g)$ is projectively related to $\left(M, g^{\prime}\right), \lambda$ is homothetic on $M$. It follows from lemma 1 that either $\lambda$ is identically zero on $M$ (and hence $\nabla=\nabla^{\prime}$ on $M$ ) or $\lambda$ cannot vanish at any point of $U$ (since Riem does not vanish at any point of $U$ ). This argument improves on that in [9] which may now be used to show the following results [11]. In the event that $(M, g)$ is of holonomy type $R_{11}$ each point of $U$ admits a coordinate neighbourhood with coordinates $u, v, x^{3}, x^{4}$ on which $\partial / \partial u$ is $g$-null and $\nabla$-covariantly constant and $g$ and $g^{\prime}$ take the following forms. For $g$ one has (with Greek indices taking the values 3 and 4)

$$
d s^{2}=2 d u d v+u^{2} h_{\alpha \beta}\left(x^{3}, x^{4}\right) d x^{\alpha} d x^{\beta}
$$

where $h$ represents a positive definite metric in the hypersurfaces of constant $u$ and $v$. Next one has for the 1 -form $\psi(=d \chi)$

$\psi=d \chi, \quad \chi=\frac{1}{2} \ln F, \quad F=\kappa^{4}\left[1+2 c u v+2 e_{1} u+\left(e_{1}^{2}-c e_{2}\right) u^{2}\right]^{-1}$ 
and for the metric $g^{\prime}$, projectively related to $g$,

$$
\begin{aligned}
d s^{\prime 2}=\kappa F g- & \kappa^{-3} F^{2}\left(c v^{2}+2 e_{1} v+e_{2}\right) d u^{2} \\
& -\kappa^{-3} F^{2} c u^{2} d v^{2}-\kappa^{-3} F^{2} 2 u\left(c v+e_{1}+\left(e_{1}^{2}-c e_{2}\right) u\right) d u d v
\end{aligned}
$$

for a positive constant $\kappa$ and constants $c, e_{1}$ and $e_{2}$ with $c$ as in (14). The metric in (17) can be tidied up by going to new coordinates $U, V, x^{3}, x^{4}$. In the case that $c \neq 0$ this change is given by $u=U(1-c U V)^{-1}$, $v=\left(c e_{2} U+2 c V-2 e_{1}-U\left(e_{1}-c V\right)^{2}\right)(2 c(1-c U V))^{-1}$ and the metric $g^{\prime}$ in (17) becomes, up to a constant scaling,

$$
d s^{2}=2 d U d V+c U^{2} d V^{2}+U^{2} h_{\alpha \beta}\left(x^{3}, x^{4}\right) d x^{\alpha} d x^{\beta}
$$

For the case $c=0$ the coordinate change is $u=U\left(1-e_{1} U\right)^{-1}$, $v=\left(V+e_{2} U / 2\right) /\left(1-e_{1} U\right)$ and $g^{\prime}$ in (18) becomes

$$
d s^{\prime 2}=2 d U d V+U^{2} h_{\alpha \beta}\left(x^{3}, x^{4}\right) d x^{\alpha} d x^{\beta}
$$

Thus the metric $g^{\prime}$ in the new coordinates $U, V, x^{3}, x^{4}$, for $c=0$, takes a form similar to that for $g$ in (15).

In the event that $(M, g)$ is of holonomy type $R_{10}$ or $R_{13}$ each point of $U$ admits a coordinate neighbourhood with coordinates $t, z, x^{3}, x^{4}$ on which $g$ and $g^{\prime}$ take the following forms. To deal with both simultaneously, the constants $\epsilon_{1}$ and $\epsilon_{2}$ are introduced each of which may take either of the values \pm 1 and $\partial / \partial t$ is $(\nabla-)$ covariantly constant. For $g$ one has

$$
d s^{2}=\epsilon_{1} d t^{2}+\epsilon_{2} d z^{2}+z^{2} h_{\alpha \beta}\left(x^{3}, x^{4}\right) d x^{\alpha} d x^{\beta}
$$

where $h$ represents a positive definite metric in the hypersurfaces of constant $t$ and $z$. Then $\psi$ is given by

$\psi=d \chi, \quad \chi=\frac{1}{2} \ln F, \quad F=\kappa^{4}\left[1+\epsilon_{2}\left(c+\epsilon_{1}\left(c_{2} c-c_{1}^{2}\right)\right) z^{2}+\epsilon_{1}\left(c t^{2}+2 c_{1} t+c_{2}\right)\right]^{-1}$

and the metric $g^{\prime}$, projectively related to $g$, is given by

$$
\begin{aligned}
g^{\prime}=\kappa F g-\kappa^{-3} F^{2}\left(c t^{2}+2 c_{1} t+c_{2}+\epsilon_{2}\left(c c_{2}-c_{1}^{2}\right) z^{2}\right) d t^{2} \\
-\kappa^{-3} F^{2}\left(c+\epsilon_{1}\left(c c_{2}-c_{1}^{2}\right)\right) z^{2} d z^{2}-\kappa^{-3} F^{2} 2 \epsilon_{1} \epsilon_{2}\left(c t+c_{1}\right) z d t d z
\end{aligned}
$$

where $\kappa$ is a positive constant and $c, c_{1}$ and $c_{2}$ constants with $c$ as before. To get the $R_{13}$ case one takes $\epsilon_{1}=-1$ and $\epsilon_{2}=1$ and vice-versa for the $R_{10}$ case. Again a coordinate transformation may be used to cast $g^{\prime}$ into a form similar to that for $g$ in (20). First define $q \equiv c+\epsilon_{1}\left(c c_{2}-c_{1}^{2}\right)$ and then a function $Q(T)$ by it being a solution of the differential equation $d Q / d T=1+\epsilon_{1}\left(c Q^{2}+2 c_{1} Q+c_{2}\right)$. The coordinate change is then $t=Q(T), z=Z \sqrt{\left(1+\epsilon_{1}\left(c Q^{2}+2 c_{1} Q+c_{2}\right)\left(1-\epsilon_{2} q Z^{2}\right)^{-1}\right.}$ and $g^{\prime}$ becomes, up to a constant scaling,

$d s^{\prime 2}=\epsilon_{1}\left(1-\epsilon_{2} q Z^{2}\right) d T^{2}+\epsilon_{2}\left(1-\epsilon_{2} q Z^{2}\right)^{-1} d Z^{2}+Z^{2} h_{\alpha \beta}\left(x^{3}, x^{4}\right) d x^{\alpha} d x^{\beta}$

In the event that $q=0$ this becomes, up to a constant scaling

$$
d s^{2}=\epsilon_{1} d T^{2}+\epsilon_{2} d Z^{2}+Z^{2} h_{\alpha \beta}\left(x^{3}, x^{4}\right) d x^{\alpha} d x^{\beta}
$$


For the remainder of this section $(M, g)$ is assumed to be of holonomy type $R_{9}$ or $R_{14}$. The problem of (local) projective relatedness for a space-time $(M, g)$ of one or the other of these holonomy types will be solved in the following sense. Suppose that $U$ is some non-empty, connected, open subset of $M$ and let $g^{\prime}$ be a metric on $U$ which is projectively related to (the restriction of) $g$ to $U$. First let $A, B, C, D$ and $O$ denote the subsets of $\mathrm{M}$ on which the curvature class is $\mathbf{A}, \mathbf{B}, \mathbf{C}, \mathbf{D}$ and $\mathbf{O}$, respectively. The subset $A$ is necessarily open (section 2). One then has the disjoint decompositions $M=A \cup B \cup C \cup D \cup O=A \cup \operatorname{int} B \cup \operatorname{int} C \cup \operatorname{int} D \cup \operatorname{int} O \cup F$ where int denotes the interior operator in the manifold topology on $M$ and $F$ is the closed subset of $M$ determined by the disjointness of the decomposition and can be shown to have empty interior [1]. Thus, $M \backslash F$ is open and dense in $M$. Now decompose $U$ disjointly as $U=A^{\prime} \cup B^{\prime} \cup C^{\prime} \cup D^{\prime} \cup O^{\prime}=A^{\prime} \cup \operatorname{int} B^{\prime} \cup \operatorname{int} C^{\prime} \cup \operatorname{int} D^{\prime} \cup \operatorname{int} O^{\prime} \cup F^{\prime}$ where $A^{\prime}=A \cap U$, etc and int here means the interior operator in the subspace topology of $U$ (but this is the same as the original interior operator in $M$ since $U$ is open in $M$ ). Clearly $A^{\prime}$ is open in $U$ (and also in $M$ ), int $B^{\prime}=\operatorname{int} B \cap U$, etc, and again one can show that $F^{\prime}$ has empty interior and so $U \backslash F^{\prime}$ is open and dense in $U$. If $(M, g)$ has holonomy type $R_{9}$ it can be shown that $B=B^{\prime}=\emptyset$ and that $\nabla^{\prime}=\nabla$ on $\operatorname{int} C^{\prime}$ and $\operatorname{int} D^{\prime}$ whereas if $(M, g)$ has holonomy type $R_{14}, \nabla^{\prime}=\nabla$ on $\operatorname{int} B^{\prime}$ and $\operatorname{int} C^{\prime}$ [11]. In the holonony $R_{14}$ case, the situation in $\operatorname{int} D^{\prime}$ is essentially as for the holonomy type $R_{11}$ (curvature class $\mathbf{D}$ case) described above [9]. Thus the interesting cases concern the set $A^{\prime}$.

The theory of section 3 now applies to $g$ and $g^{\prime}$ on $U$ and attention is here focussed on the open subset $A^{\prime}$ of $U$. Let $W$ be some non-empty, connected, open subset of $A^{\prime}$. If the space-time $(M, g)$ has holonomy type $R_{9}$, the (restricted) space-time $(W, g)$ can also be checked to have this holonomy type whilst, if $(M, g)$ is of type $R_{14},(W, g)$ has holonomy type $R_{9}, R_{12}$ or $R_{14}$. These results follow since $W \subset A^{\prime}$. Suppose the associated 1-form $\lambda$ vanishes on $W$ (so that $\nabla a=0$ on $W$, from (12)). It then follows from theorem 1(iv) and the comments following it that $a=\alpha g$ on $W(\alpha \in \mathbb{R})$. Theorem 2 then shows that the "trivial" solution $\lambda=0$ and $a=\alpha g$ on $U$ is the only possible solution to the problem. So it will be assumed that $\lambda$ does not vanish over any nonempty open subset of $A^{\prime}$. It then follows that if $W$ is a non-empty open, connected subset of $A^{\prime}$ on which $\lambda$ is nowhere zero, the holonomy type of $(W, g)$ is $R_{9}$ or $R_{14}$ (the $R_{12}$ possibility is ruled out by theorem 3(i) [9]). Then because $(M, g)$ has holonomy type $R_{9}$ or $R_{14}$ each point of $A^{\prime}$ admits an open neighbourhood $V$ on which there exists a nowhere-zero, null (unique up to a scaling) $\nabla$-recurrent vector field $l$ and which is hence hypersurface-orthogonal, $l_{[a ; b} l_{c]}=0$. Thus this neighbourhood may be chosen so that a scaled version of $l$ (also labelled $l$ ) exists on it and which is normal and satisfies $l_{a}=u_{, a}$ and $l_{a ; b}=\beta l_{a} l_{b}$ on $V$ for functions $u, \beta: V \rightarrow \mathbb{R}$. Now on any such open set $V$ the Ricci identity for $l$ gives $l_{d} R_{a b c}^{d}=l_{a} F_{b c}$ with $F_{a b}=2 l_{[a} \beta_{, b]}$ on $V$ (where a comma denotes a partial derivative) and since $V \subset A^{\prime}, F$ and $\beta_{, a}$ are each nowhere-zero on $V$. Now one may also assume that $V$ is chosen so that, in addition to the above, it admits a global smooth null tetrad $l, n, x, y$ based on the null vector field $l$ and then it follows that the bivectors $l \wedge x$ and $l \wedge y$ are in the kernel of the curvature map $f$ at each point 
of $V$. It then follows from lemma 1 that

$$
\lambda_{a ; b}=\rho g_{a b}+\sigma l_{a} l_{b}
$$

on $V$ for functions $\rho$ and $\sigma$ on $V$ which are easily shown to be smooth. On substituting (25) into (13) one finds

$$
a_{a e} R_{b c d}^{e}+a_{b e} R_{a c d}^{e}=\sigma\left(g_{a c} l_{b} l_{d}+g_{b c} l_{a} l_{d}-g_{a d} l_{b} l_{c}-g_{b d} l_{a} l_{c}\right)
$$

On contracting (26) with $l^{a}$ and defining $L_{a}=a_{a b} l^{b}$ (noting that it is nowhere-zero on $V$ since $a$ is non-degenerate on $V$ ) one finds that $L_{e} R_{b c d}^{e}=L_{b} F_{c d}$ on $V$. A contraction of this equation first with $L^{b}$ shows that $L$ is null and second with $l^{b}$ shows that $L^{e} l_{e} F_{a b}=0$ and hence that $L_{a} l^{a}=0$, on $V$. It follows that $L_{a}$ and $l_{a}$ are proportional on $V$ and so $a_{a b} l^{b}=\phi l_{a}$ for some clearly smooth function $\phi$ on $V$ which is nowhere zero on $V$ because of the non-degeneracy of $a$. A differentiation of this last equation and use of (12) (and $\left.l_{a ; b}=\beta l_{a} l_{b}\right)$ gives

$$
g_{a b} \lambda_{c} l^{c}+\lambda_{a} l_{b}=l_{a} \phi_{, b} \quad\left(\Rightarrow \quad \lambda_{a}=\phi_{, a}=\phi^{\prime} l_{a}\right)
$$

A rank argument and the fact that $l$ is nowhere zero on $V$ gives $\lambda_{a} l_{b}=l_{a} \phi_{, b}$ and so $\phi$ is a (nowhere-zero) function of $u$ only (and a prime denotes $d / d u$ ). Here $\phi^{\prime}$ is a smooth nowhere-zero function of $u$ on $V$. Thus the function $\rho$ in (25) vanishes on $V$.

It was assumed above that the 1 -form $\lambda$ does not vanish over any non-empty open subset of $A^{\prime}$. Thus if $A^{\prime \prime}$ denotes the open (necessarily dense) subset of $A^{\prime}$ over which $\lambda$ is nowhere zero, $\lambda$ may represent the null recurrent (unique up to a not necessarily constant scaling) vector field $l$ that arises from either of the holonomy types considered on $A^{\prime \prime}$. (Of course, choices other than $\lambda^{a}=l^{a}$ are available from (27). This problem of uniqueness is dealt with in section 6.1.) Then the function $\beta$ may be taken as that associated with $\lambda\left(\lambda_{a ; b}=\beta \lambda_{a} \lambda_{b}\right)$ on each such $V$. Now $\beta$ is defined on $A^{\prime \prime}$ and can be checked not to vanish over any non-empty open subset of $A^{\prime \prime}$. Similar remarks apply to the function $\beta_{, a} l^{a}$ and so one may restrict, for later convenience, to an open dense subset $\stackrel{*}{A}$ of $A^{\prime}$ on which $\lambda, \beta$ and $\beta_{, a} l^{a}$ are all nowhere zero. Neighbourhoods such as $V$ above will now be assumed to be subsets of $\stackrel{*}{A}$ and, since $\beta_{, a} l^{a}$ is nowhere zero on $V$ (and so the bivector $F$ is timelike on $V$ ), the tetrad $l, n, x, y$ will be assumed chosen so that $F$ is a non-zero multiple of $l \wedge n$ on $V$.

One is now in a position to write down an algebraic expression for Sinjukov's tensor $a$ on $\stackrel{*}{A}$ locally in terms of the null tetrad $l, n, x, y$. However, a problem arises because of possible changes in the Segre type of $a$ with respect to $g$ and a consequent uncertainty of the smoothness of the eigenvalues and eigenvector fields of $a$.

Such problems have been dealt with in [11] and a further, mild restriction on $V$ is necessary and will be assumed to have been made. It turns out that for holonomy type $R_{14} V$ may be chosen as a coordinate domain with coordinates $u, z, x$ and $y$ and the metric form for $g$ is [11]

$$
d s^{2}=2 d u d z+\sqrt{z} b(u) d u^{2}+u^{2} e^{2 w(x, y)}\left(d x^{2}+d y^{2}\right)
$$


for arbitrary smooth functions $b$ and $w$, and for $g^{\prime}$ it is, up to a constant scaling,

$d s^{\prime 2}=\frac{2 d u d z}{(1+c u)^{3}}+\frac{(\sqrt{z} b(u)(1+c u)-2 c z) d u^{2}}{(1+c u)^{4}}+\frac{u^{2} e^{2 w(x, y)}\left(d x^{2}+d y^{2}\right)}{(1+c u)^{2}}$

After a coordinate transformation from $u$ and $z$ to $U$ and $Z$ where $u=U /(1-c U)$, $z=Z /(1-c U), x$ and $y$ unchanged, and with $B(U) \equiv b(U /(1-c U))(1-c U)^{-3 / 2},(29)$ gives a form for $g^{\prime}$ similar to that for $g$ and is, up to a constant scaling,

$d s^{\prime 2}=2 d U d Z+\sqrt{Z} B(U) d U^{2}+U^{2} e^{2 w\left(x^{3}, x^{4}\right)}\left(d\left(x^{3}\right)^{2}+d\left(x^{4}\right)^{2}\right)$

If the holonomy type is $R_{9}$ one gets for the metrics $g$ and $g^{\prime}$ either (28)-(30) with $w$ a harmonic function $\left[\partial^{2} w / \partial\left(x^{3}\right)^{2}+\partial^{2} w / \partial\left(x^{4}\right)^{2}=0\right]$ or, in coordinates $u, z, x, y$ on $V$, for $g[11]$

$$
d s^{2}=2 d u d z+\sqrt{z} b(u) d u^{2}+u^{2} d x^{2}+(u-E)^{2} d y^{2}
$$

where $E=D-C=$ constant and with corresponding metric $g^{\prime}$ given by, up to a constant scaling,

$d s^{\prime 2}=\frac{2 d u d z}{(1+c u)^{3}}+\frac{(\sqrt{z} b(u)(1+c u)-2 c z) d u^{2}}{(1+c u)^{4}}+\frac{u^{2} d x^{2}}{(1+c u)^{2}}+\frac{(u-E)^{2} d y^{2}}{(1+c E)(1+c u)^{2}}$

After a coordinate transformation given by $u=\mu U /(1-c \mu U), z=\mu Z /(1-c \mu U)$, $y=Y / \mu$, where $\mu=\sqrt{1+c E}), B(U)=b(\mu U /(1-c \mu U))(1-c \mu U)^{-3 / 2}$ and $E^{\prime}=E / \mu^{3}$ this becomes, up to a constant scaling,

$$
d s^{\prime 2}=2 d U d Z+\sqrt{Z} B(U) d U^{2}+U^{2} d x^{2}+\left(U-E^{\prime}\right)^{2} d Y^{2}
$$

In all cases, the 1 -form $\psi$ is a nowhere-zero multiple of $u_{a}$ on $V$. It is remarked here that the ambiguity in the choice of $\lambda$ contained in (27) and mentioned earlier is fully accounted for in (29), (30), (32) and (33) (see section 6.1). It is also remarked that that the metrics (28)-(30) are of type $D$ in the Petrov classification, as are (31)-(33), provided $E=0$. The metrics (31)-(33) with $E \neq 0$ are of Petrov type $I I$.

\section{Holonomy Type Preservation under Projective Relationship}

Suppose a space-time $\left(M, g^{\prime}\right)$ is locally projectively related to a space-time $(M, g)$ on an open subset $U \subset M$. Then, if $U$ is not dense in $M$, there is little one can say regarding the relationship between the holonomy types of $(M, g)$ and $\left(M, g^{\prime}\right)$ except that the holonomy type of $(M, g)$ must reflect any holonomy restriction forced upon it by the holonomy type of $(U, g)$ which, in turn, is conditioned by the holonomy type of $\left(U, g^{\prime}\right)$. But considerable freedom remains in the class of metrics on $M$ which restrict to $g$ on $U$. Since finding metrics locally projectively related to flat metrics is, in principle, straightforward, the assumption will be made that $(M, g)$ is non-flat.

Another problem arises as to the effect of the assumption of non-flatness in the study of projective relatedness. Suppose $(M, g)$ and $\left(M, g^{\prime}\right)$ are projectively related and let $O \subset M$ be the closed subset of $M$ consisting of all points of $M$ at which the curvature, 
Riem, from $\nabla$ vanishes. If $\operatorname{int} O=\emptyset,(M, g)$ is non-flat so suppose that $U \equiv \operatorname{int} O$ is not empty. Then if $V$ is a (necessarily connected, open) component of $U$ the (restricted) space-times $(V, g)$ and $\left(V, g^{\prime}\right)$ are projectively related and, since $(V, g)$ is flat, $\left(V, g^{\prime}\right)$ is of constant curvature [14]. It then follows that either $\left(V, g^{\prime}\right)$ is flat, and so $\left(M, g^{\prime}\right)$ is not non-flat, or the rank of the curvature map $f$ associated with $\nabla^{\prime}$ is 6 at each point of $V$ and so the holonomy type of $\left(V, g^{\prime}\right)$, and hence of $\left(M, g^{\prime}\right)$, is $R_{15}$. The following lemma is thus proven.

Lemma 2 Suppose $(M, g)$ and $\left(M, g^{\prime}\right)$ are globally projectively related and neither is of holonomy type $R_{15}$. Then if one of them is non-flat, so also is the other (equivalently, if $(M, g)$ is non-flat then either $\left(M, g^{\prime}\right)$ is also non-flat or $(M, g)$ has holonomy type $\left.R_{15}\right)$.

Now suppose a space-time $\left(M, g^{\prime}\right)$ is (globally) projectively related to a space-time $(M, g)$. If $(M, g)$ has holonomy type $R_{2}, R_{3}, R_{4}, R_{6}, R_{7}, R_{8}$ or $R_{12}$, then $\nabla=\nabla^{\prime}$ and the holonomy type of $\left(M, g^{\prime}\right)$ is obviously the same as that of $(M, g)$. This section examines the possibilities for the holonomy type of $\left(M, g^{\prime}\right)$ when $(M, g)$ is non-flat and of one of the other holonomy types. It is convenient here to note that if $\tilde{M}$ is the universal covering manifold for $M$ with projection $\pi: \tilde{M} \rightarrow M$ one has a metric $\tilde{g}$ on $\tilde{M}$ which is the pullback of $g$ under $\pi, \tilde{g}=\pi^{*} g$. Then $(\tilde{M}, \tilde{g})$ and $(M, g)$ are locally isometric, under the isometric immersion, $\pi$. It then follows that the restricted holonomy groups of $(M, g)$ and $(\tilde{M}, \tilde{g})$ are isomorphic [17]. But since $\tilde{M}$ is simply connected, the holonomy group of $(\tilde{M}, \tilde{g})$ coincides with its restricted holonomy group and so the holonomy group of $(\tilde{M}, \tilde{g})$ is connected and its holonomy algebra coincides with that of $(M, g)$. In addition, if $\tilde{\nabla}$ is the Levi-Civita connection associated with $\tilde{g}$, the existence of the map $\pi$ shows that any geodesic in $(\tilde{M}, \tilde{g})$ projects, under $\pi$, to one in $(M, g)$. It also shows that if $\tau$ is a geodesic in $(M, g)$ passing through $m \in M$ and if $\tilde{m} \in \tilde{M}$ is such that $\pi(\tilde{m})=m$ there is a unique geodesic $\tilde{\tau}$ in $(\tilde{M}, \tilde{g})$ passing through $\tilde{m}$ such that $\pi(\tilde{\tau})=\tau$. It follows that $(M, g)$ and $\left(M, g^{\prime}\right)$ are projectively related if and only if $(\tilde{M}, \tilde{g})$ and $\left(\tilde{M}, \tilde{g}^{\prime}\right)$ are, where $\tilde{g}^{\prime}=\pi^{*} g^{\prime}$. Since the set of points where their respective Riemann tensors vanish coincide under $\pi,(\tilde{M}, \tilde{g})$ is non-flat if and only if $(M, g)$ is. Thus certain problems concerning projective relatedness can be dealt with by considering simply connected space-times and this simplifies the situation as far as holonomy groups are concerned.

Now suppose that $(M, g)$ is non-flat and of holonomy type $R_{9}$. Then so also is the covering $(\tilde{M}, \tilde{g})$ and hence $\tilde{M}$ admits a global $\tilde{\nabla}$-recurrent null vector field $\tilde{l}$ (since the holonomy group of $(\tilde{M}, \tilde{g})$ is connected (see, e.g. $[17,1])$ ). Suppose that $\left(M, g^{\prime}\right)$ is projectively related to $(M, g)$ and let $\tilde{g}^{\prime}=\pi^{*} g^{\prime}$ so that $(\tilde{M}, \tilde{g})$ and $\left(\tilde{M}, \tilde{g}^{\prime}\right)$ are projectively related. Let $\tilde{\lambda}$ and $\tilde{a}$ be a solution pair of (12) with associated 1-form $\tilde{\psi}$ and potential $\tilde{\chi}$ so that $\tilde{\psi}=d \tilde{\chi}$. Now, recalling the theory of such holonomy types described in section 4 , one may disjointly decompose with respect to the connection $\nabla$ and in the notation of that section, $M=A \cup \operatorname{int} C \cup \operatorname{int} D \cup F$ with $M \backslash F$ open and dense in $M$. Similarly, and in an obvious corresponding notation, one may write with respect to $\tilde{\nabla}$, $\tilde{M}=\tilde{A} \cup \operatorname{int} \tilde{C} \cup \operatorname{int} \tilde{D} \cup \tilde{F}$ with $\tilde{M} \backslash \tilde{F}$ open and dense in $\tilde{M}$. It follows that $\pi(\tilde{A})=A$, $\pi(\tilde{C})=C, \pi(\operatorname{int} \tilde{C})=\operatorname{int} C$, etc. The work of section 4 applied to $(\tilde{M}, \tilde{g})$ then shows 
that $\nabla=\nabla^{\prime}$ (that is, $\tilde{\lambda}=0$ ) on $\operatorname{int} \tilde{C} \cup \operatorname{int} \tilde{D}$. Thus if $\tilde{A}$ is empty $\nabla=\nabla^{\prime}$ on $M$ and so $\left(M, g^{\prime}\right)$ has holonomy type $R_{9}$. If $\tilde{A}$ is not empty it admits an open dense subset each point of which admits an open coordinate neighbourhood $V \subset \tilde{A}$ on which the metric $g$ takes the form (28) or (31) and in either case $\tilde{\psi}_{a}$ and $\tilde{\lambda}_{a}$ are nowhere zero and proportional to $\tilde{l}_{a}$. On $V$, (10) implies that $\tilde{\lambda}^{a}\left(=g^{a} b \tilde{\lambda}_{b}\right)=-e^{2 \tilde{\chi}} \tilde{\psi}^{\prime a}$, where $\tilde{\psi}^{\prime a}=\tilde{g}^{\prime a b} \psi_{b}$. Thus $\tilde{\lambda}^{a}, \tilde{l}^{a}, \tilde{\psi}^{a}$ and $\tilde{\psi}^{\prime a}$ are each nowhere-zero and proportional (and hence $\tilde{g}$-null) on $V$. Now $\tilde{l}$ is $\tilde{\nabla}$-recurrent on $V$ and, since $\tilde{l}^{a} \tilde{\psi}_{a}=0,(7)$ gives (with a semi-colon denoting a $\tilde{\nabla}$-covariant derivative and a stroke denoting a $\tilde{\nabla}^{\prime}$-covariant derivative)

$$
\tilde{l}^{a}{ }_{b b}-\tilde{l}_{; b}^{a}=\tilde{l}^{c}\left(\delta_{c}^{a} \tilde{\psi}_{b}+\delta_{b}^{a} \tilde{\psi}_{c}\right)=\tilde{l}^{a} \tilde{\psi}_{b}
$$

Thus $\tilde{l}$ is $\tilde{\nabla}^{\prime}$-recurrent on $V$. Since $\nabla=\nabla^{\prime}$ on $\operatorname{int} \tilde{C} \cup \operatorname{int} \tilde{D}$, $\tilde{l}$ is $\tilde{\nabla}^{\prime}$-recurrent on an open dense subset of $\tilde{M}$ and so, with $\tilde{l}_{a}^{\prime}=\tilde{g}_{a b}^{\prime} \tilde{l}^{b}$, one gets the equivalent statement to this recurrence condition $\tilde{l}_{[a}^{\prime} \tilde{l}_{b] \mid c}^{\prime}=0$ (where square brackets denote the usual skewsymmetrisation of indices) for the vector field $\tilde{l}$ over an open dense subset of $\tilde{M}$ and hence on $\tilde{M}$. It follows that $\tilde{l}$ is $\tilde{\nabla}^{\prime}$-recurrent on $\tilde{M}$. The existence of such a vector field on $\tilde{M}$ shows that the holonomy type of $\left(\tilde{M}, \tilde{g}^{\prime}\right)$ is $R_{9}, R_{10}, R_{11}, R_{13}$ or $R_{14}$ (the lower dimensional holonomy types being excluded by theorem 3 since then $(\tilde{M}, \tilde{g})$ would not have holonomy type $R_{9}$ ).

Continuing, if $\left(\tilde{M}, \tilde{g}^{\prime}\right)$ has holonomy type $R_{10}, R_{11}$ or $R_{13}$ (and recalling that $\tilde{M}$ is simply connected) it follows that $\tilde{M}$ admits a global nowhere-zero $\tilde{\nabla}^{\prime}$-covariantly constant vector field $k, \nabla^{\prime} k=0$, (and which is the only global $\tilde{\nabla}^{\prime}$-recurrent vector field on $\tilde{M}$ up to the usual scaling). Thus, since $\tilde{l}^{a}$ is $\tilde{\nabla}^{\prime}$-recurrent, from the above argument, it follows that $\tilde{l}$ and $k$ are proportional on $\tilde{M}$. Now again assuming that $\tilde{A}$ is non-empty, one returns to the neighbourhood $V$ and recalls that $\tilde{\psi^{\prime a}}$ is nowhere-zero and proportional to $\tilde{l}^{a}$ on $V$. It then follows that $\tilde{\psi}^{\prime a}$ is ( $\tilde{\nabla}^{\prime}$-recurrent and) proportional to $k$ on $V$, so that, $k^{a}=\alpha \tilde{\psi^{\prime a}}$ on $V$ for $\alpha: V \rightarrow \mathbb{R}$ with $\alpha$ smooth and nowhere zero on $V$. Thus $\left(\alpha \tilde{\psi^{\prime a}}\right)_{\mid b}=0$ on $V$. Then using (34) applied to $\alpha \tilde{\psi^{\prime a}}$ one finds that ${\tilde{\psi^{\prime}}}_{; b}=\tilde{\psi^{a}} \beta_{b}$ where the 1 -form $\beta$ is the gradient of $\gamma \equiv-(\log |\alpha|+\chi)$ on $V$. From this, $\left(e^{-\gamma} \tilde{\psi^{\prime a}}\right)_{; b}=0$ and then, from the Ricci identity, the curvature tensor Riem of $(\tilde{M}, \tilde{g})$ satisfies $\tilde{R}_{b c d}^{a}{\tilde{\psi^{\prime}}}^{d}=0$ which contradicts the fact that the $\tilde{\nabla}$-curvature class of $V$ is $\mathbf{A}$. Thus $\tilde{A}=\emptyset$ and one achieves the result that $\tilde{\nabla}^{\prime}=\tilde{\nabla}$ on $\tilde{M}$ and which contradicts the fact that $(\tilde{M}, \tilde{g})$ is of holonomy type $R_{9}$. Thus $\left(\tilde{M}, \tilde{g}^{\prime}\right)$ is of holonomy type $R_{9}$ or $R_{14}$.

That the holonomy type of $\left(\tilde{M}, \tilde{g}^{\prime}\right)$, and hence of $\left(M, g^{\prime}\right)$, is $R_{9}$ now follows from the Ambrose-Singer theorem [18] (see also [17, 1]). Working with the original space-times $(M, g)$ and $\left(M, g^{\prime}\right)$, this theorem says, roughly speaking, that the holonomy algebra of $\left(M, g^{\prime}\right)$ can be constructed by first choosing (any) $m \in M$ and, for each $m^{\prime} \in M$ and each piecewise $C^{1}$ curve $c$ from $m^{\prime}$ to $m$, computing the range space of the linear map $f$ (introduced in section 2) with respect to $\nabla^{\prime}$ at $m^{\prime}$ and parallely transporting, using $\nabla^{\prime}$, each member of it to $m$ along $c$. If this is done for each such $m^{\prime}$ and $c$, the collection of bivectors accumulated at $m$ spans the holonomy algebra of $\left(M, g^{\prime}\right)$. Now since the holonomy type of $(M, g)$ is $R_{9}$ the range space of $f$ for this space-time at any point of $A$ (is 3-dimensional and) consists of simple bivectors whose blades each contain the 
recurrent vector field evaluated at that point (from the curvature class and holonomy conditions). The same is true for all other points of $M$ (except that the bracketed condition is replaced by "is 1- or 2-dimensional"). Now the metric $g^{\prime}$ as displayed in (30) and (33) is identical in form to that for $g$ in (28) and (31) and this shows that the range of the corresponding curvature map $f^{\prime}$ for the space-time $\left(M, g^{\prime}\right)$ at any point of an open dense subset of $A$ also consists of simple bivectors whose blades each contain the direction spanned by the (common) recurrent null vector field evaluated at that point, as described above. The same is true for the points in $\operatorname{int} C$ and $\operatorname{int} D$ since $\nabla^{\prime}=\nabla$ on these subsets. It follows from a continuity argument on the range of $f^{\prime}$ together with the Ambrose Singer theorem that $\left(M, g^{\prime}\right)$ also has holonomy type $R_{9}$. It also follows from lemma 2 that $\left(M, g^{\prime}\right)$ is non-flat.

Now suppose that $(M, g)$ is non-flat, of holonomy type $R_{14}$ and that $\left(M, g^{\prime}\right)$ is projectively related to it. Then, as mentioned in section 4 , one may disjointly decompose $M$ with respect to $\nabla$ as $M=A \cup \operatorname{int} B \cup \operatorname{int} C \cup \operatorname{int} D \cup F$ where $F$ is closed with empty interior and $\nabla=\nabla^{\prime}$ on int $B \cup \operatorname{int} C$. If int $D$ is empty the proof above in the $R_{9}$ case shows that, in the first instance, $\left(M, g^{\prime}\right)$ has holonomy type $R_{9}$ or $R_{14}$ and finally that it is $R_{14}$, otherwise a contradiction is obtained from the main result of the previous paragraph. So suppose that int $D$ is not empty. If $A$ is not empty the above proof still reveals that the holonomy type of $\left(M, g^{\prime}\right)$ cannot be $R_{n}$ for $n \in\{2,3,4,6,7,8,9,10,11,12,13\}$ and so must be $R_{14}$ or $R_{15}$. This result also holds if $A$ is empty. To see this note that the possibilities $R_{n} n \in\{2,3,4,6,7,8,12\}$ for $\left(M, g^{\prime}\right)$ are ruled out as before and $R_{9}$ is ruled out by the argument of the previous paragraph. So suppose that $\left(M, g^{\prime}\right)$ has holonomy type $R_{10} R_{11}$ or $R_{13}$. Then for $m \in \operatorname{int} D$ there is an open neighbourhood $V \subset \operatorname{int} D$ of $m$ and a $\nabla^{\prime}$-covariantly constant, nowhere-zero vector field $k$ on $V$ and which, from the Ricci identity for $k$, anihilates the associated curvature tensor $\operatorname{Riem}^{\prime}$ on $V, R^{\prime a}{ }_{b c d} k^{d}=0$. Also, $V$ may be chosen so as to admit a nowhere zero, $g$-null, $\nabla$-recurrent vector field $l$ for $(M, g)$ and which, since $(M, g)$ has holonomy type $R_{14}$, can be shown to anihilate its associated curvature tensor on $V,\left(R_{b c d}^{a} l^{d}=0\right)[9]$. Then (9) contracted with $k^{c} l^{d}$ gives at each $m \in V k^{a}\left(\psi_{b c} l^{c}\right)=l^{a}\left(\psi_{b c} k^{c}\right)$ and so either (a) $k$ and $l$ are proportional at $m$ or (b) $\psi_{b c} c^{c}=\psi_{b c} k^{c}=0$ at $m$. If (a) holds then a contraction of (9) with $k^{d}$ easily leads to $\psi_{a b}=0$ at $m$ whilst if (b) holds a contraction of (9) with $k^{b}$ leads to $R_{b c d}^{a} k^{b}=0$ and similarly one achieves $R_{b c d}^{\prime a} l^{b}=0$. Another contraction of (9) with $k^{d}$ finally gives $\psi_{a b}=0$ at $m$. So in either case, $\psi_{a b}$ vanishes on $V$ and hence on int $D$. However, $\nabla=\nabla^{\prime}$ on $\operatorname{int} B \cup \operatorname{int} C$ and so, on this latter subset, Riem ${ }^{\prime}=$ Riem and (9) then easily shows that $\psi_{a b}=0$ on $\operatorname{int} B \cup \operatorname{int} C$. It follows, since $A$ is empty, that $\psi_{a b}=0$ on an open dense subset of $M$ and hence on $M$. Recalling the definition of $\psi_{a b}$, one sees that, for $(M, g), \psi_{a ; b}=\psi_{a} \psi_{b}$ and so $\left(e^{-\psi} \psi_{a}\right)_{; b}=0$. Then using (7) one gets $\psi_{a \mid b}=-\psi_{a} \psi_{b}$ and so $\left(e^{\psi} \psi_{a}\right)_{\mid b}=0$. Thus, either $\psi \equiv 0$ on $M\left(\Rightarrow \nabla=\nabla^{\prime}\right.$ on $\left.M\right)$ or $(M, g)$ admits a global, nowhere zero, covariantly constant vector field. Since $\left(M, g^{\prime}\right)$ is assumed of holonomy type $R_{10} R_{11}$ or $R_{13}$, each of these contradicts the assumed $R_{14}$ holonomy type of $(M, g)$ and so the desired result follows. Thus if $(M, g)$ is of holonomy type $R_{14},\left(M, g^{\prime}\right)$ must be of holonomy type $R_{14}$ or $R_{15}$. Again, lemma 2 shows that $\left(M, g^{\prime}\right)$ is non-flat. Further, 
and as mentioned earlier, $\left(M, g^{\prime}\right)$ can only be of holonomy type $R_{15}$ if int $D$ is not empty.

Finally suppose that $(M, g)$ is non-flat and of holonomy type $R_{10}, R_{11}$ or $R_{13}$. Then from earlier remarks and the work of the previous paragraph, $\left(M, g^{\prime}\right)$ cannot be of holonomy type $R_{n}$ for $n \in\{2,3,4,6,7,8,9,12,14\}$ and so must be of holonomy type $R_{10}, R_{11}, R_{13}$ or $R_{15}$. One can say a little more here. In fact, if $\left(M, g^{\prime}\right)$ is not of type $R_{15}$, then for each $m \in M$ there is an open neighbourhood $V$ of $m$ and two vector fields $p$ and $q$ on $V$ which are covariantly constant with respect to $\nabla$ and $\nabla^{\prime}$, respectively. Thus, from the Ricci identities on $p$ and $q, R_{b c d}^{a} p^{b}=0 R_{b c d}^{\prime a} q^{b}=0$ on $V$. Similar contractions to those used in the previous paragraph give $\psi_{a b}=0$ on $V$ and hence on $M$. Thus, as seen above, $e^{-\psi} \psi_{a}$ and $e^{\psi} \psi_{a}$ are global covariantly constant (co)vector fields on $M$ for $\nabla$ and $\nabla^{\prime}$, respectively. So either $\psi=0\left(\left(\Rightarrow \nabla=\nabla^{\prime}\right)\right.$ or $\left(M, g^{\prime}\right)$ and $\left(M, g^{\prime}\right)$ are each, somewhat curiously, forced to admit a global nowhere-zero, covariantly constant vector field. Put another way, if $(M, g)$ and $\left(M, g^{\prime}\right)$ are projectively related with each being one of the holonomy types $R_{10}, R_{11}$ or $R_{13}$ and with one of them not admitting a global covariantly constant vector field, the projective relatedness is trivial.

Again suppose that each of the above projectively related space-times $(M, g)$ and $\left(M, g^{\prime}\right)$ is of one of the holonomy types $R_{10}, R_{11}$ or $R_{13}$. Suppose, in addition, that $(M, g)$ of type $R_{11}$. Then from the previous paragraph $M$ admits a global $\nabla$-covariantly constant, $g$-null (co)vector field $e^{-\chi} \psi$ which is a global gradient (of $-e^{-\chi}$ ). It follows that either $\psi \equiv 0$ on $M$ (and so $\nabla^{\prime}=\nabla$ and $\left(M, g^{\prime}\right)$ is also of holonomy type $R_{11}$ ) or $\psi$ is nowhere zero on $M$ and hence that, in some coordinate neighbourhood $V$ of $M$, $\psi$ is nowhere-zero and $g$ and $g^{\prime}$ are related as in (15)-(19). Further, in the notation of these equations, since the gradient of $e^{-\chi}$ is $\nabla$-covariantly constant, $\chi$ and hence $F$ is a function only of the coordinate $u$. It follows that the constant $c=0$ and that, from (19), the (global, nowhere-zero, $\nabla^{\prime}$-covariantly constant) (co)vector field $e^{\chi} \psi$ on $M$ is $g^{\prime}$-null on $V$ and hence on $M$. Thus $\left(M, g^{\prime}\right)$ has holonomy type $R_{11}$. If, on the other hand, $(M, g)$ of type $R_{13}, M$ admits a global $\nabla$-covariantly constant, timelike (co)vector field $e^{-\chi} \psi$ which is a global gradient (of $-e^{-\chi}$ ). Again, if $\psi=0, \nabla=\nabla^{\prime}$. Otherwise, an inspection of the metric $g$ in (20) and (21) shows that $F$ must be a function only of $t$ and hence that $q=c+\epsilon_{1}\left(c_{2} c-c_{1}^{2}\right)=0$. Then the form for the metric $g^{\prime}$ in (24) on the open subset $V$ reveals that the global covariantly constant vector field admitted by $\left(M, g^{\prime}\right)$ must also be timelike on $V$ and hence on $M$ and so $\left(M, g^{\prime}\right)$ must have holonomy type $R_{13}$ and be of curvature class D. Similar comments apply, from (20)-(24), to the holonomy type $R_{10}$.

The following theorem has thus been established.

Theorem 4 Let $(M, g)$ and $\left(M, g^{\prime}\right)$ be projectively related space-times. Then

(i) If $(M, g)$ is of holonomy type $R_{2}, R_{3}, R_{4}, R_{6}, R_{7}, R_{8}$, or $R_{12}$, or is of holonomy type $R_{10}, R_{11}$ or $R_{13}$ and there exists $m \in M$ such that the curvature rank at $m$ is $\geq 2$ then $\left(\nabla=\nabla^{\prime}\right.$ and $)(M, g)$ and $\left(M, g^{\prime}\right)$ have the same holonomy type.

(ii) If $(M, g)$ has holonomy type $R_{9}$ and is non-flat, then $\left(M, g^{\prime}\right)$ has holonomy type $R_{9}$ and is non-flat. 
(iii) If $(M, g)$ has holonomy type $R_{14}$ and is non-flat with its curvature class $\mathbf{D}$ region having empty interior, $\left(M, g^{\prime}\right)$ has holonomy type $R_{14}$ and is non-flat. If the restriction on the region $\mathbf{D}$ is dropped, $\left(M, g^{\prime}\right)$ has holonomy type $R_{14}$ or $R_{15}$ and is non-flat.

(iv) If $(M, g)$ has holonomy type $R_{10}$ (respectively, $R_{11}$ or $\left.R_{13}\right)$ and is non-flat, $\left(M, g^{\prime}\right)$ has holonomy type $R_{10}$ (respectively, $R_{11}$ or $R_{13}$ ) or $R_{15}$ and is non-flat.

(v) If $(M, g)$ has holonomy type $R_{15},\left(M, g^{\prime}\right)$ has holonomy type $R_{9}, R_{10}, R_{11}, R_{13}$, $R_{14}$ or $R_{15}$ and cannot be of type $R_{9}$ if it is non-flat.

It is remarked that a consideration of the metrics (18) and (23) shows that the possibility of $\left(M, g^{\prime}\right)$ being $R_{15}$ in part (iv) above can occur.

\section{Some Concluding Remarks}

\subsection{Uniqueness results}

In this section a useful observation is pointed out which shows that, in the holonomy type $R_{9}$ case and (with a certain clause inserted) the holonomy type $R_{14}$ case studied here, any two "non-trivial" solutions $(a, \lambda)$ and $(\tilde{a}, \tilde{\lambda})$ of $(25)$ are, at least locally, tightly related. Returning to the discussion following (25) and recalling how, for either of these two holonomy types, one chose the special open neighbourhood $\stackrel{*}{A} \subset A$ suppose that $W$ is an connected open subset of $\stackrel{*}{A}$ on which $l, \lambda$ and $\tilde{\lambda}$ are nowhere zero, $l=d u, \lambda=l$ and $\tilde{\lambda}=\tilde{\phi} \lambda$ where $\tilde{\phi}$ is a nowhere-zero function of $u$ only, on $W, \lambda_{a ; b}=\beta \lambda_{a} \lambda_{b}, \tilde{\lambda}_{a ; b}=\sigma \tilde{\lambda}_{a} \tilde{\lambda}_{b}$ for nowhere zero functions $\beta$ and $\sigma$, where $\tilde{\phi}^{2} \sigma=\tilde{\phi}^{\prime}+\tilde{\phi} \beta$, on $W$ and $\beta_{, a} l^{a}$ and $\sigma_{, a} l^{a}$ are nowhere zero on $W$. Further, if $a$ and $g$ are proportional over some non-empty open subset of $W,(12)$, after some obvious contractions, reveals the contradiction that $\lambda=0$ on this subset. Thus one may work on an open dense subset $W^{\prime}$ of $W$ on which, in addition to the above conditions, one also has that $a$ and $g$ are nowhere proportional on $W^{\prime}$. Then, by comparing the corresponding versions of (13) for the solutions $(a, \lambda)$ and $(\tilde{a}, \tilde{\lambda})$ of $(12)$ on $W^{\prime}$ one finds that the combination $f=\tilde{a}-\mu a$ where $\mu \equiv \frac{\sigma \tilde{\phi}^{2}}{\beta}$ satisfies $f_{e(a} R_{b) c d}^{e}=0$ on $W^{\prime}$ and hence, since the curvature is of class $\mathbf{A}$ on $\stackrel{*}{A}$, theorem 1(iv) gives on $W^{\prime}$

$$
\tilde{a}-\mu a=\nu g
$$

for some smooth function $\nu$ on $W^{\prime}$. Then taking the covariant derivative of (35) and substituting the corresponding versions of (12) for $a$ and $\tilde{a}$, one has

$$
(\tilde{\phi}-\mu)\left(g_{a c} l_{b}+g_{b c} l_{a}\right)=a_{a b} \mu_{, c}+g_{a b} \nu_{, c}
$$

Still on the set $W^{\prime}$, if at some point of it (and hence in some neighbourhood of this point) $(\tilde{\phi}-\mu)$ does not vanish, contract (36) firstly with $l^{a}$ and secondly with $g^{a b}$ to obtain two equations from which one can deduce that both $d \nu$ and $d \mu$ are proportional to $l$ at $m$ and hence that $\nu$ and $\mu$ are, in this neighbourhood, functions of at most $u$. A contraction of (36) with $l^{c}$ then gives a contradiction and so $(\tilde{\phi}-\mu)=0$ on $W^{\prime}$. Then 
(36) together with the above remarks about the tensors $a$ and $g$ shows that $d \mu$ and $d \nu$ vanish on $W^{\prime}$ and hence on $W$ and with $\mu$ nowhere zero on $W$. Since $W$ is connected, $\nu$ and $\mu$ are constants on $W$ with $\mu \neq 0$. The conclusion, from (35) and (12) applied to $(a, \lambda)$ and $(\tilde{a}, \tilde{\lambda})$, is that $\tilde{\lambda}=\mu \lambda$ on $W$. Thus if one solution pair $(a, \lambda)$ of $(12)$ exists on $W$, the only others that may occur satisfy $\tilde{\lambda}=c \lambda$ and hence, from (12), $\nabla(\tilde{a}-\mu a)=0$. The curvature class $\mathbf{A}$ assumption (theorem 1(iv)) then shows that $\tilde{a}=\mu a+d g$ for some constant $d$ and so $(\tilde{a}, \tilde{\lambda})$ is of the form $(\mu a+d g, \mu \lambda)$. [This should be compared to the uniqueness situation in such holonomy types for finding projective symmetries [19]]. This argument covers the situation for both holonomy types $R_{9}$ and $R_{14}$ on (an open dense subset of) $A$. Again for both these types, and on the subsets int $C$ and int $D$ of $M$, one achieves the result $\nabla^{\prime}=\nabla$ and the relationship between $g$ and $g^{\prime}$ is easily found from holonomy theory $[1,9]$. For the holonomy type $R_{14}$ and the subset int $D$ the situation is more complicated.

\subsection{Generic Space-Times}

If one puts the Whitney $C^{\infty}$ topology on the set $\mathcal{L}_{M}$ of all smooth Lorentz metrics on $M$ there exists an open dense subset, $\mathcal{L}^{\prime}{ }_{M}$, of $\mathcal{L}_{M}$ (that is, of space-time structures on $M)$ in this topology, each member of which has the property that its curvature tensor has rank at least 4 at each point of $M[20]$ (see also [1]). A space-time $(M, g)$ will be called generic if $g \in \mathcal{L}^{\prime}{ }_{M}$ and, if $U$ is a non-empty, connected, open subset of $M,(U, g)$ will be called a generic region of $M$ if the restriction of $g$ to $U$ is in $\mathcal{L}^{\prime}{ }_{U}$. It is then clear from section 2 that no generic space-time or generic region of any space-time may contain any covariantly constant type $(0,2)$ symmetric tensor field other than constant multiples of the metric $g$ since $M$ (or $U$ ) must be of curvature class $A$. It is noted here that if $(M, g)$ is generic then, whether or not $(U, g)$ is a generic region of $M$, the curvature rank is at least 4 over $U$ and the above remarks about covariantly constant type $(0,2)$ symmetric tensor fields still hold on $M$ and $U$.

Now suppose $(M, g)$ is a space-time and $U$ is a non-empty, connected, open subset of $M$ such that $U$ is a generic region of $M$. One then has the following results, part (i) of which may be regarded as a type of "rigidity" result for $\lambda$ (in the sense that if $\nabla=\nabla^{\prime}$ over some "sufficiently general" subset $U$ of $M$ then $\nabla=\nabla^{\prime}$ on $M$ ). It is convenient for their statements to introduce a definition regarding the vector space $\mathrm{S}$ introduced in section 3. If $(a, \lambda),(b, \mu) \in S$, write $(a, \lambda) \sim(b, \mu)$ if $b=a+\alpha g$ on $M(\alpha \in \mathbb{R})$ (and hence, from (12), $\lambda=\mu$ on $M)$. It is easily checked that $\sim$ is an equivalence relation on $S$. If $V$ is an open subset of $M$, one may speak of the "restriction" of $\sim$ to $V$ in terms of solutions of (12) defined on $V$, in an obvious way.

Theorem 5 Let $(M, g)$ be a space-time and $(U, g)$ a generic region of $M$.

(i) If $(a, \lambda),(b, \mu) \in S$ and $\lambda=\mu$ on $U$ then $\lambda=\mu$ on $M$ and $(a, \lambda) \sim(b, \mu)$ on $M$. In particular, if $(a, \lambda) \in S$ and $\lambda=0$ on $U$, then $a=\alpha g(\alpha \in \mathbb{R})$ and $\lambda=0$, on $M$. 
(ii) Let $(a, \lambda) \in S$ and let $\left(a_{U}, \lambda_{U}\right)$ denote the restriction (in an obvious sense) of $(a, \lambda)$ to $U$. If $\left(b^{\prime}, \mu^{\prime}\right)$ is a solution of (12) on $U$ and $\left(b^{\prime}, \mu^{\prime}\right) \sim\left(a_{U}, \lambda_{U}\right)$ on $U$ then $\left(b^{\prime}, \mu^{\prime}\right)$ is extendible to $(b, \mu) \in S$ and $(a, \lambda) \sim(b, \mu)$ on $M$. Said another way, if two solution pairs of (12) on $U$ are related under $\sim$ on $U$ and one is extendible to being a member of $S$ then so is the other and these extensions are then related under $\sim$ on $M$.

\section{Proof}

(i) If $\lambda=\mu$ on $U$ then $\nabla(b-a)=0$ on $U$. Since $U$ is a generic region of $M$, $b-a=\alpha g$ on $U(\alpha \in \mathbb{R})$ and so $(a+\alpha g, \lambda) \in S$ and $(a+\alpha g, \lambda)=(b, \mu)$ on $U$ and hence on $M$ by theorem 2. But $(a+\alpha g, \lambda) \sim(a, \lambda)$ and the first result follows. The second part is similar.

(ii) If $\left(a_{U}, \lambda_{U}\right) \sim\left(b^{\prime}, \mu^{\prime}\right)$ on $U$ then $\lambda_{U}=\mu^{\prime}$ on $U$ and since $U$ is a generic region of $M, b^{\prime}=a_{U}+\alpha g$ on $U$. Then $(a+\alpha g, \lambda) \in S$ and $(a+\alpha g, \lambda)=\left(b^{\prime}, \mu^{\prime}\right)$ on $U$ and $(a+\alpha g, \lambda) \sim(a, \lambda)$ on $M$. So $(b, \mu) \equiv(a+\alpha g, \lambda)$ is the desired extension.

\subsection{Projective Symmetry}

A global smooth vector field $X$ on a space -time $(M, g)$ is called projective if each local flow diffeomorphism $\phi_{t}$ associated with $X$ and with open domain $U$ and range $\phi_{t}(U)$ maps (unparametrised) geodesics of $U$ to (unparametrised) geodesics of $\phi_{t}(U)$ each with respect to (the restriction to $U$ of) $\nabla$. Then $X$ is called affine if it is projective and if, in addition, each map $\phi_{t}$ preserves affine parameters. If $X$ is projective but not affine it is called proper projective. If $X$ is proper projective, there exists a non-empty open subset $U \subset M$ on which a local flow $\phi_{t}$ is defined and which does not preserve affine parameters. Then the metrics $g$ and $\phi_{t}^{*} g$ can be seen to be locally (but non-trivially) projectively related on $U$. This shows that if a proper projective vector field exists on $(M, g)$ then $\nabla$ is locally (non-trivially) projectively related to some other (Levi-Civita) connection $\nabla^{\prime}$ on some open subset $U$. The converse of this result is false. In fact, a comparison of [6] and [21] reveals examples of FRWL space-times admitting (locally) non-trivially projectively related (FRWL) metrics but with no proper projective vector fields. (Related references on projective structure and FRWL metrics are [22] and [23].) Another example is the metric (28) which always admits non-trivially projectively related metrics (29) but only admits a proper projective symmetry for particular choices of the function $b(u)$ [19]. The above result on local flows shows that if $(M, g)$ satisfies either (i) it has one of the holonomy types $R_{2}, R_{3}, R_{4}, R_{6}, R_{7}, R_{8}$ or $R_{12}$, and is non-flat or, (ii) it has one of the holonomy types $R_{10}, R_{11}$ or $R_{13}$ and has curvature rank greater than 1 at each $m \in M$, then the induced geometry on any connected open subset $U$ of $M$ also has one of these properties and theorem 3 then shows that any projective vector field on $M$ is affine. (cf [19]).

Expressed in terms of the Lie derivative $L_{X}$, the situation is that any proper projective vector field $X$ with associated projective 1-form $d p$ gives rise to a Sinjukov solution to $(12)$ of the form $\left(a \equiv L_{X} g-2 p g, \lambda=d p\right)$, with the arbitrary constant in $p$ 
chosen such that $a$ is non-singular. The lack of a converse is due to there being, in general, no guarantee of the existence of a vector field $X$ satisfying $L_{X} g=a+2 l g$ for a given Sinjukov solution $(a, \lambda)$ where $\lambda=d l$ for some smooth function $l$ on $M$.

\section{References}

[1] Hall G.S., Symmetries and Curvature Structure in General Relativity. World Scientific, (2004).

[2] Petrov A.Z., Einstein Spaces, Pergamon, (1969).

[3] Hall G.S. and Lonie D.P., The principle of equivalence and projective structure in spacetimes, Class. Quant. Grav 24 (2007), 3617.

[4] Hall G.S. and Lonie D.P, Projective equivalence of Einstein spaces in general relativity Class. Quant. Grav 26 (2009),125009.

[5] Kiosak V. and Matveev V., Complete Einstein metrics are geodesically rigid, Comm. Math. Phys. 289, (2009) 383.

[6] Hall G.S. and Lonie D.P., The principle of equivalence and cosmological metrics, J. Math. Phys. 49 (2008), 022502.

[7] Mikes J., Hinterleitner I. and Kiosak V.A., On the Theory of geodesic Mappings of Einstein Spaces and their Generalizations, in the Albert Einstein Centenary International Conference, ed J.-M Alini and A Fuzfa, American Institute of Physics, (2006).

[8] Mikes J., Kiosak V. and Vanzurova A., Geodesic Mappings of manifolds with affine connection (2008)

[9] Hall G.S. and Lonie D.P., Holonomy and Projective Equivalence in 4-Dimensional Lorentz Manifolds, Sigma 5, (2009), 066.

[10] Schell J.F., Classification of Four-Dimensional Riemannian Spaces, J. Math. Phys. 2 (1961), 202.

[11] Hall G.S. and Lonie D.P. Projective Structure and Holonomy in four-dimensional Lorentz manifolds. Journal of Geometry and Physics (2010) doi:10.1016/j.geomphys.2010.10.007

[12] Levi-Civita T., Sulle trasformazioni delle equazioni dinamiche, Ann. Mat. Milano 24, (1886), 255.

[13] Thomas T.Y., Differential Invariants of Generalised Spaces, Cambridge, (1934).

[14] Eisenhart L.P., Riemannian Geometry, Princeton, (1966).

[15] Sinyukov N.S., Geodesic Mappings of Riemannian Spaces (in Russian), "Nauka", Moscow, MR0552022, Zbl 0638.53020, (1979).

[16] Hall G.S. and Rendall A.D., Local and global algebraic structures in general relativity, Int. J. Theor. Phys. 28, (1989), 365.

[17] Kobayashi S. and Nomizu K., Foundations of Differential Geometry vol 1, Interscience, New York, (1963)

[18] Ambrose W. M. and Singer I. M., Trans Am Math Soc 75, (1953), 428.

[19] Hall G.S. and Lonie D.P. Class. Quant. Grav 21, (2004), 4549.

[20] Rendall A.D. J. Math. Phys 29, (1988), 1569.

[21] Hall G.S. Projective symmetry in FRW space-times. Class Quant Grav. 17, (2000), 4637.

[22] Nurowski P., Is dark energy meaningless? arXiv:1003.1503 [math.DG]

[23] Gibbons G.W. and Warnick C.M., Dark energy and projective symmetry. Physics Letters B 688, (2010), 337 . 